\title{
Calvarial Tuberculosis Mimicking Meningioma: An Unusual Cause for Aphasia with Scalp Swelling
}

\author{
Raghunath A. ${ }^{1}$ Biju Bhadran ${ }^{1}$ Krishnakumar P. ${ }^{1}$ Sathi P. P. ${ }^{2}$ \\ ${ }^{1}$ Deparment of Neurosurgery, Government T. D. Medical College, \\ Alappuzha, Kerala, India \\ 2 Department of Pathology, Government T. D. Medical College, \\ Alappuzha, Kerala, India \\ Address for correspondence Raghunath A, MCh, Department of \\ Neurosurgery, Government T. D. Medical College, Alappuzha, Kerala \\ 688005, India (e-mail: raghunimhans@yahoo.co.in). \\ Indian J Neurosurg 2016;5:136-139.
}

\begin{abstract}
Background Calvarial tuberculosis is uncommon. It usually presents as a punched out lesion with varying extend of extradural and subgaleal collection. However, typical imaging findings may be lacking and may appear as a neoplasm-like meningioma. This report details the clinical and imaging findings of a left frontal lesion which mimicked neoplasm.

Clinical Presentation A 54-year-old female patient presented with decreased speech output and a gradually growing swelling on the left side of the scalp for 1 year duration. On imaging, lesion was left frontal subgaleal plane and left frontal parenchyma with different signal changes. Bony changes were minimal. Our preoperative diagnosis was a central nervous system tumor-like convexity meningioma with an extracranial growth or a dural-based sarcoma or slow-growing metastasis. The patient underwent left frontal craniotomy and excision of the lesion. Histopathology was suggestive of tuberculous granuloma.

Keywords

- calvarial tuberculosis

- CNS tuberculosis

- meningioma mimic

Conclusion Our report represented a case of diffuse variety of calvarial tuberculosis with significant growth into brain parenchyma which is rare. It should be kept as one of the differential diagnosis of lesions with intracranial and scalp component. Awareness will help in managing and prognosticating these lesions.
\end{abstract}

\section{Introduction}

Central nervous system (CNS) tuberculosis (TB) can present as meningitis, cerebritis, tuberculoma, or tubercular abscess. Tuberculous osteitis of skull is an uncommon manifestation. It can present with fluctuant swelling in the scalp with discharging sinuses superficially or with significant extradural collection or meningitis in a deeper aspect. Parenchymal tuberculomas are also a less common form of CNS TB and can mimic CNS neoplasms such as meningioma. ${ }^{1,2}$ Misdiagnosis is common because of the rarity of the disease and overlapping imaging findings. The present report is a case of CNS TB with significant involvement of calvarium and parenchyma and appeared as an extra-axial dural-based neoplasm. Radiological findings and literature review are detailed.

\section{Case Report}

The patient is a 54-year-old lady of low socioeconomic status who was kept in an old age home due to financial constraints and personality changes. She was put on antipsychotic drugs (quetiapine). She developed decreased speech output for 12 months duration and gradually growing swelling on the received

November 12, 2015

accepted

December 18, 2015

published online

June 8, 2016
DOI http://dx.doi.org/

$10.1055 / \mathrm{s}-0036-1581978$

ISSN 2277-954X (c) 2016 Neurological Surgeons' Society
of India

License terms

(®) $\ominus \circledast$ 
left side of the scalp for 8 month duration. On examination, she was conscious, obeying, ambulant, but depressed in affect with decreased attention span and features of Broca aphasia. Other than gross cognitive dysfunction and aphasia, she was not having any significant motor deficits or meningeal signs. Fundus did not show papilledema. The scalp swelling was firm, painless, size of $3 \times 4 \mathrm{~cm}$, without any skin changes was in the left frontal region just behind the hairline. Regional lymph nodes were not palpably enlarged. There were no other swellings or organomegaly.

Laboratory tests revealed mild microcytic hypochromic anemia with a hemoglobin level of $9.4 \mathrm{~g} / \mathrm{dL}$, erythrocyte sedimentation rate of $100 \mathrm{~mm} / 1 \mathrm{st}$ hour. Screening for metastasis did not reveal the presence of primary lesion elsewhere.

Cranial computed tomography showed isodense lesion in left frontal subgaleal plane and left frontal parenchyma with perilesional edema. Bone windows did not show any overt hypertrophy/hyperostosis or gross osteolysis between intracranial and scalp component of the lesion. However, there were mild changes in the form of speckled erosion of the outer cortex of bone visualized only in single $10-\mathrm{mm}$ axial cut ( - Fig. 1A, B) On magnetic resonance imaging (MRI) signal intensities were different for the intracranial lesion and extracranial part. Intracranial lesion was T1 hypo- to isointense T2 minimally hyperintense with restricted diffusion characteristics and significant vasogenic perilesional edema. On the other hand, the latter showed T1 hypointensity,
T2 hyperintensity, and restricted diffusion characteristics. On contrast intracranial part showed intense, but nonuniform heterogeneous enhancement with dural tail extending more posteriorly and extracranial part showed peripheral enhancement (-Fig. 1C-E). Fine-needle aspiration cytology from the scalp lesion was inconclusive.

With these clinical and imaging findings, initial diagnoses were CNS tumor-like convexity meningioma with an extracranial growth or a dural-based sarcoma or slowgrowing metastasis. Second, we also considered extracranial part to be an unrelated scalp swelling-like sebaceous cyst because of relatively intact bone and a different signal and contrast characteristics on MRI. Metastatic workup did not reveal the presence of a primary elsewhere or hematologic malignancy.

The patient underwent left frontal craniotomy and excision of the lesion. Extracranial component was subgaleal in plane and consisted of yellowish cheesy partially liquefied tissue. In the outer cortex of bone under the swelling, changes were noted in an area of approximately $1 \mathrm{~cm}$ diameter. Intracranial component was yellowish solid, nonsuckable, and mildly vascular. Inner cortex showed a wider area of adherence and changes ( - Fig. 2A, B). The lesion was eroding the dura and extending into the frontal parenchyma with ill-defined arachnoid plane superficially and gliotic plane at deeper part. Histopathology revealed typical tuberculous granulomas with areas of caseation necrosis and Langhans giant cells (-Fig. 2C, D).
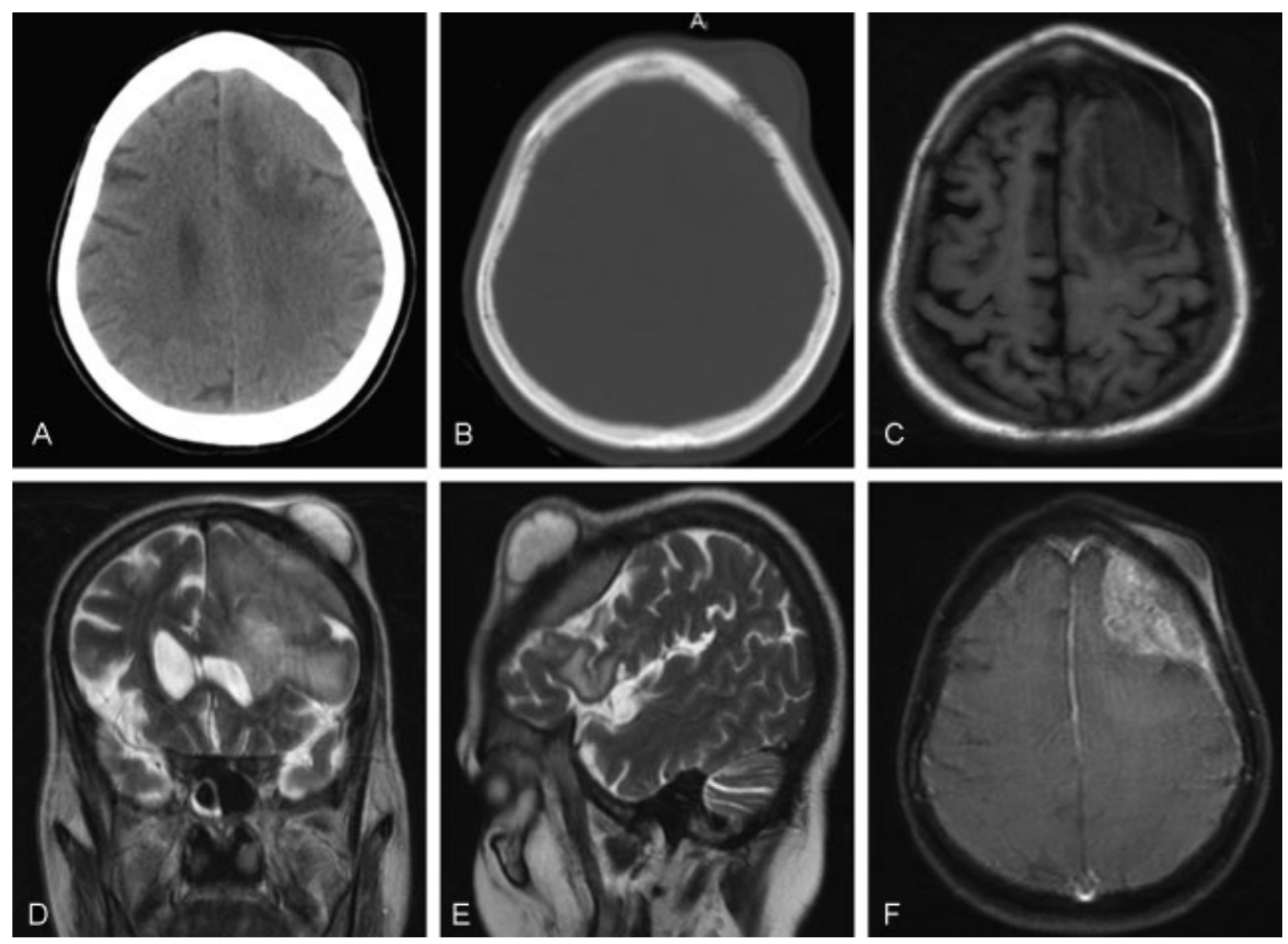

Fig. 1 Imaging characteristics of lesion. (A) Appearance in plain axial CT head, the lesion is almost isodense. (B) Bone windows showing a small area of erosion of outer cortex. (C) On MRI T1W image shows hypo- to isotense lesion left frontal. (D and E) T2W coronal and sagittal image showed the isotense lesion left frontal with cerebral edema and mass effect. But the extracranial part is showing hyperintensity, (F) on contrast lesion is showing heterogeneous enhancement. CT, computed tomography; MRI, magnetic resonance imaging; T1W, T1-weighted; T2W, T2weighted. 

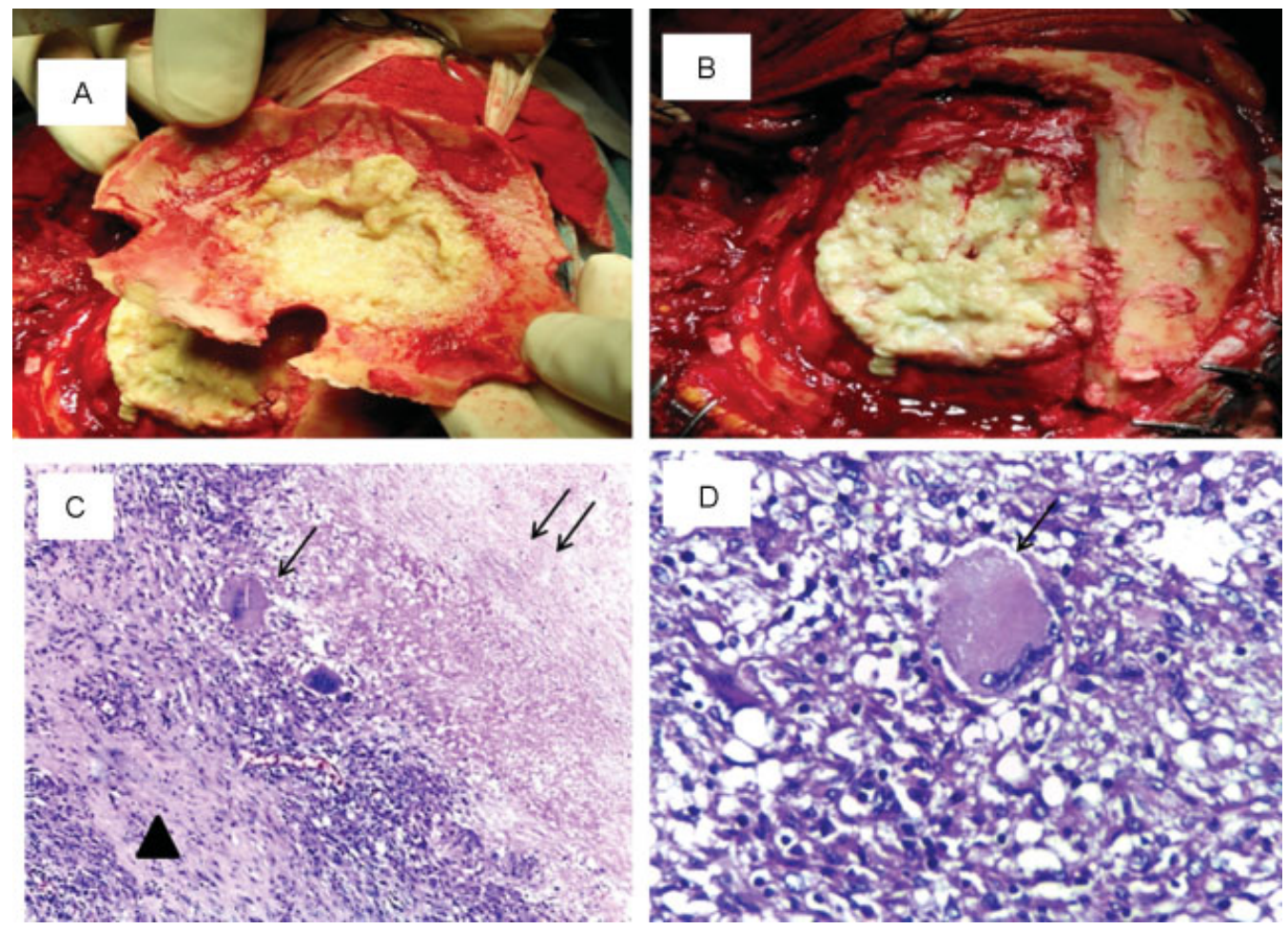

Fig. 2 Intraoperative and histopathological findings (A) involvement of an inner cortex of bone. (B) Lesion invading through dura (C) low-power view $(\times 10)$ lesion shows granuloma with giant cells (arrow), caseating necrosis (double arrow), and involvement of meninges (arrow head). (D) High-power view $(\times 40)$ central giant cell is Langhans multinucleate giant cell typical of tuberculosis (arrow).

Postoperative course in the hospital was uneventful. The patient made steady improvement with alleviation of aphasia. She became friendly with others and conversant.

\section{Discussion}

Neurotuberculosis constitute $1 \%$ of all TB, but also the most severe form extrapulmonary $\mathrm{TB}$ and meningitis is the most common form. ${ }^{3,4}$ Involvement of cranial bones is rare and forms only 0.2 to $1.3 \%$ of the cases of skeletal TB, which in turn constitute $1 \%$ cases among tubercular disease spectrum. ${ }^{5,6}$ Tuberculous osteomyelitis is usually seen in young people and commonly affect the frontal and parietal bones because of the higher amount of cancellous bone and diploic structure where tubercle bacilli appear to lodge. ${ }^{6,7}$ Our patient is a 54-years lady from poor socioeconomic background without any previous history of contact with TB.

The most common form of calvarial TB is a solitary punchedout defect called perforating TB.,8 A second type with the diffuse destruction of inner table with extradural granulation tissue is called "diffuse variety." It rarely breaches the dura and also rarely produce neurological manifestations. ${ }^{6,7}$ We believe our report represented a rare case of diffuse variety because of breach of dural barrier and significant growth into the brain parenchyma. Also, it is unlikely that nidus originated as cerebral tuberculoma (which is more common to find) and eroded into the dural, skull, and scalp.

According to MRI findings, intracranial tuberculomas are classified into three types: Noncaseating, caseating with a solid center, and caseating with a cystic center. Noncaseating granulomas exhibit hypointensity on both T1-weighted and T2-weighted imaging with uniform enhancement on contrast. Solid caseating granuloma appears isointense or hypointense on T1-weighted and T2-weighted imaging with rim enhancement and surrounding edema. With central liquefaction appear as a hypertense lesion with peripheral hypointense rim with edema on T2-weighted imaging and ring enhancement on contrast. ${ }^{2,9,10}$ Extracranial part of lesion in the present study was showing $\mathrm{T} 2$ hyperintensity suggestive of central liquefaction and imaging appearance of intracranial part is close to solid caseating granuloma without liquefaction. Similar findings were observed during surgical resection. However, because of the absence of overt osteomyelitic-like bony changes and solid-like appearance of intracranial lesion we have not suspected TB before, on imaging, or during surgery.

Summarizing, though TB is a well-established entity for pachymeningeal thickening and calvarial osteomyelitis, a significant growth of the pathology with a solid consistency into parenchyma may be easily overlooked as a CNS neoplasm, because of decreased awareness of the entity. Awareness of this entity will help in management. Since undernourished population is prone to this illness, key to prevention lies in improving nutritional and socioeconomic conditions.

\section{Conflict of Interest}

The authors report no conflict of interest. The authors alone are responsible for the content and writing of the article. This study has not received any financial support. 


\section{Acknowledgment}

The authors thank Dr. G. Harison, Senior Resident, Department of Neurosurgery, and Dr. C. Girija, Resident, Department of Pathology, for their help in preparing the article.

\section{References}

1 Boutarbouch M, Arkha Y, Gana R, El Maquili MR, Bellakhdar F. Tuberculoma of the cavernous sinus mimicking a meningioma: case report and review of the literature. J Neurol Sci 2009;278; $(1-2): 123-126$

2 Sonmez G, Ozturk E, Sildiroglu HO, et al. MRI findings of intracranial tuberculomas. Clin Imaging 2008;32(2):88-92

3 Gupta RK, Kumar S. Central nervous system tuberculosis. Neuroimaging Clin N Am 2011;21(4):795-814, vii-viii

4 DeLance AR, Safaee M, Oh MC, et al. Tuberculoma of the central nervous system. J Clin Neurosci 2013;20(10):1333-1341

5 García-García C, Ibarra V, Azcona-Gutiérrez JM, Oteo JA. Calvarial tuberculosis with parenchymal involvement. Travel Med Infect Dis 2013;11(5):329-331

6 Mukherjee KK, Kaushik R, Nada R, Khosla VK, Khandelwal N, Kak VK. Calvarial tuberculosis. Surg Neurol 2002;57(3):195-202, discussion 202-203

7 Tyagi AK, Kirollos RW, Kang NV. Tuberculous osteitis of the skull: a case report and review of the literature. Br J Neurosurg 1996; 10(4):399-401

8 Ramdurg SR, Gupta DK, Suri A, Sharma BS, Mahapatra AK. Calvarial tuberculosis: Uncommon manifestation of common disease-a series of 21 cases. Br J Neurosurg 2010;24(5):572-577

9 Saini J, Gupta RK, Jain KK. Intracranial infections: key neuroimaging findings. Semin Roentgenol 2014;49(1):86-98

10 Patkar D, Narang J, Yanamandala R, Lawande M, Shah GV. Central nervous system tuberculosis: pathophysiology and imaging findings. Neuroimaging Clin N Am 2012;22(4):677-705 